to immunosuppressants. For patients with Churg-Strauss syndrome, high initial doses of corticosteroids should be gradually reduced as the patient improves. Finally, cyclophosphamide should be used for patients with a high risk of death.

The authors highlighted the lack of highquality, easily comparable evidence, and concluded that future research should concentrate on promising new immunosuppressants and biological agents such as rituximab and infliximab.

Original article Bosch X et al. (2007) Treatment

of antineutrophil cytoplasmic antibody-associated vasculitis: a systematic review. JAMA 298: 655-669

\section{Cost-effectiveness of management strategies for renal artery stenosis}

Researchers have used a decision analytic model to evaluate the costs, benefits and cost-effectiveness ratios of five strategies for the diagnosis and management of renal artery stenosis (RAS): digital subtraction angiography (DSA; comprising diagnostic DSA and subsequent revascularization on a different day); magnetic resonance angiography (revascularization scheduled if scan results positive); CT angiography (revascularization scheduled if scan results positive); immediate tentative percutaneous revascularization (every patient with suspected RAS referred for percutaneous revascularization, with diagnostic DSA immediately before the procedure to determine whether it should go ahead); and medical therapy (antihypertensive medication, without diagnostic imaging or revascularization). Data were extracted from the Netherlands-based Renal Artery Diagnostic Imaging Study in Hypertension (RADISH) and published literature.

For a 50-year-old male with diastolic blood pressure $>95 \mathrm{mmHg}$ and at least one clinical sign of renovascular hypertension, the immediate tentative revascularization strategy cost the least $(€ 54,415)$ and was the most effective (yielding 12.265 quality-adjusted life years [QALYs]); the authors recommend this strategy in all males with suspected RAS. This strategy cost more and yielded more QALYs in a 50-year-old female with the same characteristics, but the incremental cost-effectiveness ratio when compared with DSA was $€ 7,143$ per QALY, a figure the group believes is generally acceptable.

Use of a more invasive diagnostic technique became more justified as the probability of RAS increased. Whereas immediate tentative revascularization is cost-effective in patients with high suspicion for renovascular hypertension, in patients at low risk, and in those in whom RAS is unlikely, CT angiography and medical therapy, respectively, seem to be cost-effective.

Original article van Helvoort-Postulart D et al. (2007) Renal artery stenosis: cost-effectiveness of diagnosis and treatment. Radiology 244: 505-513

\section{Stoke comorbidity grade predicts survival in ESRD patients on non-dialytic treatment}

Non-dialytic treatment (NDT) is a recognized strategy chosen by some patients with endstage renal disease (ESRD), but there are few data on the prognosis of NDT patients in terms of hospitalization rates and survival.

Wong et al. report on 3 years' experience of a dedicated multidisciplinary team clinic for NDT patients at the Royal Liverpool University Hospital, UK. The team comprised a nephrologist, a renal dietician, an anemia specialist nurse, a ward link nurse, a social worker, a community nephrology nurse and a complementary therapist. At each visit (occurring at intervals of between 1 week and 3 months), patients were reviewed by all team members. Patients were visited at home when possible.

The study included 73 patients approaching ESRD who were referred to the clinic (median age 79 years; range 40-93 years). Over 3 years, 43 patients had no hospital admissions; 58 hospital admissions (more than half for nonrenal causes) occurred among the other 30 patients. Overall, 28 patients died, 20 of these at home. Median overall survival was 1.95 years. Stoke comorbidity grade (SCG; a scoring system for predicting survival of patients on renal replacement therapy, based on the number of comorbidities) was an independent predictor of mortality, with a hazard ratio of 2.53 for each incremental increase in SCG $(P=0.005)$. There were nonsignificant trends towards shorter survival 